\title{
Noncooperative Admission Control for Differentiated Services in IEEE 802.11 WLANs
}

\author{
Yu-Liang Kuo*, Eric Hsiao-Kuang $\mathrm{Wu}^{\dagger}$, and Gen-Huey Chen* \\ * Dept. of Computer Science and Information Engineering, National Taiwan University, Taipei, Taiwan, R.O.C. \\ ${ }^{\dagger}$ Dept. of Computer Science and Information Engineering, National Central University, Chung-Li, Taiwan, R.O.C.
}

\begin{abstract}
Recent developments in wireless LAN (WLAN) standardizations have been successful to offer high-speed data services. Hence, a variety of types of traffic must be accommodated in future WLAN environments. IEEE 802.11 working group has been developing a new distributed MAC, called enhanced distributed function (EDCF), to support service differentiation in the IEEE 802.11 MAC protocol. Besides, highspeed WLAN environments are also expected to provide wireless Internet services in hot spots such as airports, parks, and etc. Since there are usually multiple service providers competing for providing wireless network access in hot spots, mobile users are free to choose their own service providers. For a specific service provider, more flows are admitted to transmit in its coverage range, more revenue (e.g., utility or profit) is gained. However, admitting many flows may make the wireless medium overloaded and degrade the QoS satisfactions of ongoing flows since the wireless medium in IEEE 802.11 is share and collision-based. Hence some mobile users may leave the current service provider and subscribe to wireless network access with another service provider. In this paper, we analyze the resource management problem in the competitive environment, in which EDCF protocol is implemented in all access points (APs) and mobile stations. We formulate admission control as a game and prove the game owns a Nash equilibrium solution. Based on the game, a service provider not only fulfills most part QoS satisfactions of ongoing flows but also increases its own revenue. We evaluate the performance by means of throughput, packet delay and bandwidth violation ratio.
\end{abstract}

\section{INTRODUCTION}

With the provisioning of high-speed WLAN environments, data services (e.g., VoIP or video-conference) with different QoS requirements will be available in future WLANs. Hence, data services will be categorized into multiple traffic classes and different priorities are used to access the wireless medium. However, in the current access mechanism of IEEE 802.11, all mobile stations have the same priority to access the wireless medium. In order to satisfy multiple traffic classes with different QoS requirements, it is desired to provide service differentiation in the IEEE 802.11 standard [5].

Providing service differentiation requires a MAC protocol to support different access priorities among different traffic classes. Recently, the IEEE 802.11 working group has been developing a new distributed protocol, called EDCF, to support service differentiation in the MAC layer [3]. EDCF is an extension of the existing distributed coordination function (DCF) to support service differentiation. More details about DCF and EDCF will be presented in Section II.

Apart from the MAC service differentiation, high-speed
WLAN environments are also expected to provide public wireless Internet services in "hot spots" such as airports, parks and etc., where a high density of mobile users will create demand for wireless network access [10]. Hence multiple wireless service providers dispose their infrastructures, e.g., APs, in hot spots to provide wireless network access. Mobile users that intend to access wireless Internet services are charged by prepaid cards, online purchasing, or other charging mechanisms. Since there are usually multiple service providers in a local region, mobile users are free to choose their own service providers. Admitting more flows to transmit in the coverage range of an AP (belonging to a certain service provider) will gain more revenue for the service provider. However, admitting many flows may make the wireless medium overloaded and degrade the QoS satisfactions of ongoing flows since the wireless medium in IEEE 802.11 is share and collision-based. Hence some mobile users may leave the current service provider and subscribe to wireless network access with another service provider.

In this paper, we analyze the resource management problem in the competitive environments, in which EDCF is implemented in access points (APs) and mobile stations. We formulate admission control as a game, which is nonzero-sum and noncooperative. Based on the game, a service provider not only fulfills most part of QoS satisfactions of ongoing flows in its radio coverage but also increases its own revenue.

The rest of this paper is organized as follows. Section II reviews the DCF and EDCF protocols. Section III suggests an admission control game formulation under which it owns a Nash equilibrium solution. Section IV shows the performance evaluation for the admission control. Section V concludes this paper.

\section{DCF AND EDCF}

DCF operates based on carrier sense multiple access with collision avoidance (CSMA/CA). A mobile station that intends to transmit a packet first senses the channel. If the channel is idle for a time period of DCF interframe space (DIFS), it can immediately start transmission. Otherwise, it generates a backoff counter. The counter starts decrement if the channel is sensed idle for a time period of DIFS. Then the counter continues to decrease until the channel is busy or the counter counts down to zero. If the channel is busy, the decrement will pause and resume after another idle time period of DIFS. When the counter counts down to zero, the mobile 
TABLE I

FOUR ACS SPECIFIED IN THE IEEE 802.11E

\begin{tabular}{ccccc}
\hline & $\mathrm{AC}_{0}$ & $\mathrm{AC}_{1}$ & $\mathrm{AC}_{2}$ & $\mathrm{AC}_{3}$ \\
\hline Values of AIFSN & 2 & 1 & 1 & 1 \\
Values of $\mathrm{CW}_{\min }$ & 32 & 32 & 16 & 8 \\
Values of $\mathrm{CW}_{\max }$ & 1024 & 1024 & 32 & 16 \\
\hline
\end{tabular}

station starts transmission. In order to avoid channel capture, a mobile station has to wait a random backoff time between two consecutive packet transmissions, even if the channel is idle for a time period of DIFS.

The backoff counter is randomly assigned a value from the range $[0, \mathrm{CW}-1]$, where $\mathrm{CW}$ is the contention window. Initially, let $\mathrm{CW}=\mathrm{CW}_{\min }$, the minimum contention window. When the transmission (or retransmission) fails, the value of $\mathrm{CW}$ is doubled until it reaches the maximum $\mathrm{CW}_{\max }=2^{m} \mathrm{CW}_{\min }$, where $m$ is called maximum backoff stage.

DCF employs two access mechanisms for packet transmission. One is two-way handshaking and the other is fourway handshaking. For the former, an ACK (acknowledgement) message is used to indicate that the transmitted packet has been correctly received by the destination station. For the later, an RTS (request-to-send) message is first sent by the source station. When the destination station receives the RTS, it replies a CTS (clear-to-send) message. After receiving the CTS message, the source station is allowed to transmit a packet. Finally, the destination station informs the source station of a successful transmission by replying an ACK message.

RTS and CTS messages carry information about the identifiers of the source and destination stations and the duration for transmitting the packet. Once hearing the RTS or CTS message, any other station will update its NAV (network allocation vector), which records the duration when the channel is busy, and defer its access to the channel.

In the IEEE 802.11 standard [1], the four-way handshaking is used to reduce the collision time when the size of the transmitted data packet is greater than a predefined length, i.e., RTSThreshold. Otherwise, the two-way handshaking is used. The four- way handshaking can also avoid the hidden terminal problem.

EDCF, which is an enhanced version of DCF, can provide a distributed access mechanism to support service differentiation in IEEE 802.11. EDCF introduces the concept of access categories (ACs). Traffic classes belonging to different ACs use different values of $\mathrm{CW}_{\min }, \mathrm{CW}_{\max }$, and arbitration interframe spacing number (AIFSN) to contend the channel. There are four ACs specified in IEEE 802.11e as shown in Table I, where the $802.11 \mathrm{~b}$ physical layer [2] is used.

EDCF requires that a mobile station has to wait a time period of AIFS before transmitting a packet or generating a backoff counter. Let $T_{A I F S}$ and $T_{S I F S}$ denote the lengths of AIFS and short IFS (SIFS), respectively. $T_{A I F S}$ is computed as follows: $T_{A I F S}=T_{S I F S}+$ AIFSN $\times \delta$, where AIFSN $\geq 1$ and $\delta$ is the length of a time slot. A traffic class with smaller
AIFSN has smaller $T_{A I F S}$ and hence has a higher probability of seizing the channel.

EDCF is backward compatible with DCF. A mobile station without EDCF has to wait at least a time period of DIFS before it can transmit a packet. Recall that IEEE 802.11 set $T_{D I F S}$, the length of DIFS, to be $T_{S I F S}+2 \delta$. A mobile station with EDCF has a higher probability of seizing the channel than those without EDCF, if it sets AIFSN=1.

\section{AdMisSion CONTROL GAME Formulation}

In this section, we formulate admission control as a twoplayer game. The admission control game is formed when a user sends a flow request to an AP. In the following subsections, we first present the system model. Second, we review the basic concepts of two-player game. Third, we present the admission control game formulation. Fourth, an important ratio - QoS satisfaction ratio - used in the game is presented. Finally, we will prove that the game owns a Nash equilibrium solution.

\section{A. System Model}

The system environment we consider is within a local region in which multiple APs compete for providing wireless network access. We assume that different APs in the local region are belonging to different service providers. In the competitive environment, mobile users are free to choose an AP for wireless Internet services. A mobile user may leave the current AP and subscribe to wireless network access with another service provider according to the degree of QoS satisfaction for the current quality.

In order to support service differentiation, EDCF protocol is implemented in all APs and mobile stations. Suppose that there are $K$ traffic classes with distinct QoS requirements in the system, where $K \geq 1$. We assume that each mobile user has packets of the same traffic class. For convenience, we refer to a mobile user that has traffic class $k$ packets as a class- $k$ user. We use $n_{k}$ to denote the number of class- $k$ users currently in the system, where $0 \leq k \leq K-1$. The term $r_{k}$ is referred to as the data rate for traffic class $k$.

\section{B. Preliminaries on Two-Player Game}

In a two-player game [8], each player has a set of strategies. A configuration is a form of a pair of strategies, in which one strategy comes from one player and the other strategy comes from the other player. Associated with each configuration, i.e., a pair of two strategies, there are two payoffs, one for each player. A payoff is a number that reflects the desirability of a configuration to a player. For a noncooperative game, each player makes a decision independently and in a manner maximizes its own payoff without knowing the decisions of other players.

Specifically, a two-player non-cooperative game has two players $p$ and $q$. We assume there are $m$ and $n$ strategies for $p$ and $q$, denoted as $\left\{u_{i}: 1 \leq i \leq m\right\}$ and $\left\{v_{j}: 1 \leq j \leq n\right\}$, respectively. Hence the configurations of the game are denoted by $\left\{u_{i}, v_{j}\right\}$, where $1 \leq i \leq m, 1 \leq j \leq n$. The payoffs for $p$ 
and $q$ are defined as two payoff matrices $P=\left[p_{i j}\right]_{m \times n}$ and $Q=\left[q_{i j}\right]_{m \times n}$. The rows of $P$ and $Q$ matrices represent the set of strategies for $p$ while the columns represent the set of strategies for $q$. In other words, $p_{i j}\left(q_{i j}\right)$ represents the payoff for $p(q)$ as $p$ chooses strategy $i$ and $q$ chooses strategy $j$, where $1 \leq i \leq m$ and $1 \leq j \leq n$.

A game can be also classified into a zero-sum and a nonzero-sum game. Specifically, if $p_{i j}+q_{i j}=0$ for all $1 \leq i \leq m$ and $1 \leq j \leq n$, it is a zero-sum game; otherwise, it is a nonzero-sum game. The number of payoff matrices for a zero-sum two-player game is one since the payoff matrix for one player can be easily obtained from the other player. Note that if $p_{i j}+q_{i j}$ are constant, it is also zero-sum.

In a two-player noncooperative nonzero-sum game, a configuration $\left\{u_{i}^{*}, v_{j}^{*}\right\}$ is said to be a Nash equilibrium solution if the values of payoff for $p$ and $q$ under $\left\{u_{i}^{*}, v_{j}^{*}\right\}$ are maximal. In other words, no player can change its strategy to get a better payoff.

It has been shown that a two-player non-cooperative nonzero-sum game may or may not have a Nash equilibrium solution [4]. However, in the proposed admission control game, we will show that it always has at least one Nash equilibrium solution, which will be presented in Section III-E.

\section{Game Formulation}

The proposed admission control game in the system is noncooperative in nature. In addition, the game is also nonzerosum. It is noted that a zero-sum game has the property that the increment for one particular payoff value will cause the decrement of the corresponding payoff value so that the summation can be zero. If a new user sends a flow request to an AP in under-loading conditions, admitting the new flow will not degrade the ongoing flows and will gain revenue for the AP. Also, the admitted user will also feel satisfied and gain its revenue. That is, both the values of payoffs for the AP and the mobile user are increasing. Therefore, the admission control game is nonzero-sum.

The two players for the admission control game are as follows:

- $p$ : the current serving AP (service provider) of the class- $k$ user;

- $q$ : a class- $k$ user that intends to initiate a new flow.

The player $p$ has the set of strategies $\left\{u_{1}, u_{2}\right\}$, where $u_{1}$ is to admit the flow request and $u_{2}$ is to reject the flow request. Similarly, the player $q$ has the set of strategies $\left\{v_{1}, v_{2}\right\}$, where $v_{1}$ is to leave the current serving AP and subscribe to another one and $v_{2}$ is to stay at the current AP.

We now define the payoff matrices $P=\left[p_{i j}\right]_{2 \times 2}$ for $p$, which is given as

$$
P=\left[\begin{array}{cc}
R+\left(R_{k}-L\right)-L_{k} & R+\left(R_{k}-L\right) \\
R-L_{k} & R
\end{array}\right] .
$$

The notations used in $P$ are defined as follows.

- $R$ : the sum of revenues for the current ongoing flows;

- $R_{k}$ : the revenue obtained by admitting a class- $k$ user;

- $L_{k}$ : the revenue loss obtained when a class- $k$ user leaves;
- $B_{k}$ : the bandwidth violation ratio for a class- $k$ user. $B_{k}$ is defined as

$$
B_{k}=\left\{\begin{array}{lll}
0 & \text { if } & \rho_{k} / n_{k}-r_{k}>0 \\
\frac{r_{k}-\rho_{k} / n_{k}}{r_{k}} & \text { if } & \rho_{k} / n_{k}-r_{k} \leq 0
\end{array},\right.
$$

where $\rho_{k} / n_{k}$ is the average available bandwidth for a class- $k$ user. The bandwidth violation ratio is zero whenever the average available bandwidth is greater than the data rate, i.e., $\rho_{k} / n_{k}-r_{k}>0$. Otherwise, the bandwidth violation ratio is defined as $\frac{r_{k}-\rho_{k} / n_{k}}{r_{k}}$. The estimation for $\rho_{k}$ can refer to [6] and is not state here;

- $L$ : potential revenue loss obtained by admitting a class- $k$ user. When an AP admits a new flow to transmit in its coverage range, it may cause potential revenue loss for the AP. It is because that each time a new flow is allowed to transmit in the system, the system may be overloading and then the QoS satisfactions of ongoing flows will be degraded;

- $S\left(B_{k}\right)$ : the QoS satisfaction ratio that represents the degree a class- $k$ user satisfies the current quality. The satisfaction ratio is a function of bandwidth violation ratio. It is noted that $0 \leq S\left(B_{k}\right) \leq 1$. The detailed derivation for $S\left(B_{k}\right)$ will be shown in Section III-D.

- $O\left(B_{k}\right)$ : the QoS dissatisfaction ratio that represents the degree a class- $k$ user dissatisfies the current quality. $O\left(B_{k}\right)$ also represents the inclination that a class- $k$ user intends to leave the current AP. It is noted that $O\left(B_{k}\right)=$ $1-S\left(B_{k}\right)$.

The element $p_{21}$ represents that the class- $k$ user will leave the current $\mathrm{AP}$ and the current $\mathrm{AP}$ will reject the flow request. Hence the value of $p_{21}$ is simply the sum of revenues of the current ongoing flows minus the revenue loss obtained by the leaving of the class- $k$ user, i.e., $R-L_{k}$. The value of $p_{22}$ is simply $R$ since the class- $k$ user does not leave the current AP and may keep trying to request later.

The values of $p_{12}$ should consider the potential loss and hence the value of $p_{12}$ is $R+R_{k}-L$. Similarly, the value of $p_{11}$ is $R+R_{k}-L-L_{k}$. We estimate the potential loss in terms of potential departures of ongoing users. The departures stem from the QoS dissatisfaction for the current quality. For class- $k$ users, the potential revenue loss is $n_{k} O\left(B_{k}\right) L_{k}$ and the total potential revenue loss for all users is hence $L=$ $\sum_{i=0}^{K-1} n_{i} O\left(B_{i}\right) L_{i}$.

Now let us discuss the payoff matrix $Q=\left[q_{i j}\right]_{2 \times 2}$. Let $U_{k}$, $\left(V_{k}\right)$ be the revenue obtained when the flow request is admitted (rejected). Apparently, $U_{k}>V_{k}$. The payoff matrix $Q$ is given as

$$
Q=\left[\begin{array}{cc}
U_{k}-Y_{k} & U_{k}-w O\left(B_{k}\right) \\
V_{k}-Y_{k} & V_{k}-w O\left(B_{k}\right)
\end{array}\right],
$$

where $Y_{k}$ and $w$ are the revenue loss when the class- $k$ leaves the current $\mathrm{AP}$ and the multiplier factor, respectively. $Y_{k}$ may be the money need to pay for the leaving plus the money need to subscribe to wireless network access with another service provider. Other explanation for $Y_{k}$ is also possible by considering more realistic market mechanisms. 
The element $q_{11}\left(q_{21}\right)$ corresponds to the configuration that the AP admits (rejects) the flow request and the class- $k$ user leaves the current AP. Hence the value of payoff for $q_{11}\left(q_{21}\right)$ is $U_{k}-Y_{k}\left(V_{k}-Y_{k}\right)$. The elements $q_{12}$ and $q_{22}$ should minus an additional term $w O\left(B_{k}\right)$ since the user which chooses to stay at the current AP may need to tolerate the possible dissatisfaction. The term $w$ is a multiplier factor and larger than 1 .

Once the payoff matrices for $p$ and $q$ are defined, the next step is to show that if this game owns a Nash equilibrium solution or not, which will show in Section III-E. Before we show the existence of Nash equilibrium solution, we derive two important ratios - QoS satisfaction ratio and QoS dissatisfaction ratio - in the following subsection.

\section{QoS Satisfaction Ratio}

We characterize the degree of QoS satisfaction for a class$k$ user as a function of bandwidth violation ratio. That is, the degree of QoS satisfaction for a class- $k$ user depends on the quality of available bandwidth. We approximate the QoS satisfaction ratio, $S\left(B_{k}\right)$, by using the Sigmoid function [9]. The Sigmoid function has been used to characterize users' satisfaction in recent literature [7], [11]. We modify the Sigmoid function to involve user's satisfaction with respect to the bandwidth violation ratio, i.e.,

$$
S\left(B_{k}\right)=\frac{1}{1+e^{-\alpha_{k}\left(\beta_{k}-B_{k}\right)}},
$$

where $\alpha_{k}$ and $\beta_{k}$ are the steepness and the center of curve. $\alpha_{k}$ and $\beta_{k}$ are used to indicate the sensitivity of bandwidth violation and the toleration of bandwidth violation for traffic class $k$. The greater the value of $\alpha_{k}$ is, the lower sensitivity of QoS degradation for traffic class $k$ is. Fig. 1 shows the plot of QoS satisfaction ratio versus bandwidth violation ratio, where $\alpha_{k}=20,30,40$ and 50, respectively. In Fig. 1, we know that the steepness of the curve is increasing as $\alpha_{k}$ is increasing and hence the sensitivity of bandwidth violation is decreasing. Similarly, the higher value of $\beta_{k}$ is, the higher toleration for the bandwidth violation is, as depicted in Fig. 2 . Once the bandwidth violation ratio is greater than $\beta_{k}$, the degree of users' satisfaction (i.e., QoS satisfaction ratio) will be degraded dramatically.

Once $S\left(B_{k}\right)$ has been defined, the QoS dissatisfaction ratio $O\left(B_{k}\right)$ is simply given as $1-S\left(B_{k}\right)$.

\section{E. Equilibrium Solutions}

Before discussing the existence of Nash equilibrium solution, we first explain the concept of domination.

Definition 1: In a two-player non-cooperative game, a strategy $u_{i}^{*}$ dominates another strategy $u_{j}^{*}$ of $p$ if it obtains better playoff regardless of what the strategy $q$ chooses, where $1<i, j<m$.

Theorem 1: The proposed admission control game possesses at least one Nash equilibrium solution.

Proof: We consider the proof in three cases:

- $R_{k}-L>0$

In this case, $u_{1}$ dominates $u_{2}$ and hence $p$ will always

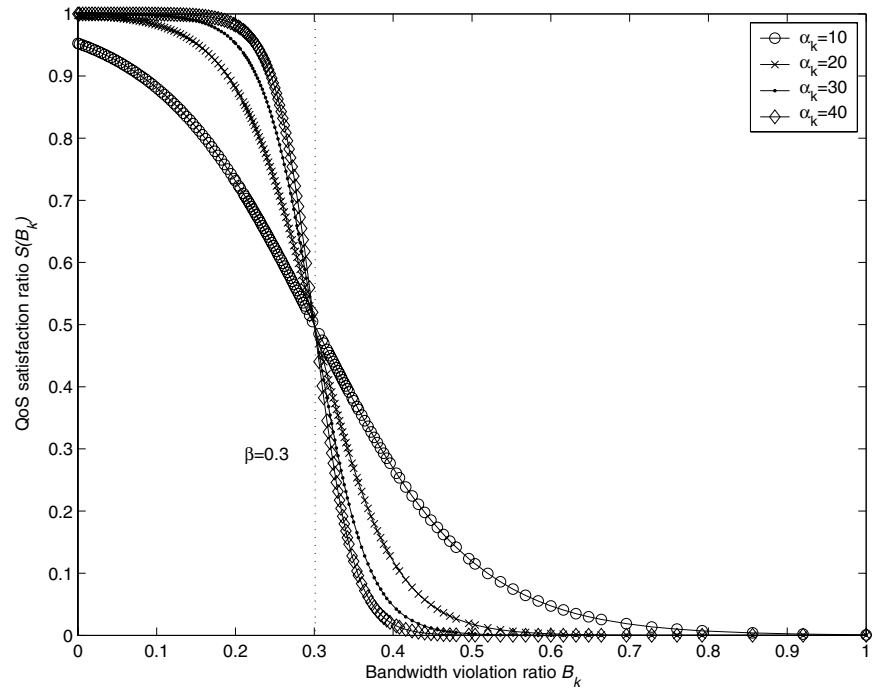

Fig. 1. QoS satisfaction ratio $S\left(B_{k}\right)$ vs. bandwidth violation ratio $B_{k}$ $\left(\alpha_{k}=10,20,30,40 ; \beta_{k}=0.3\right)$.

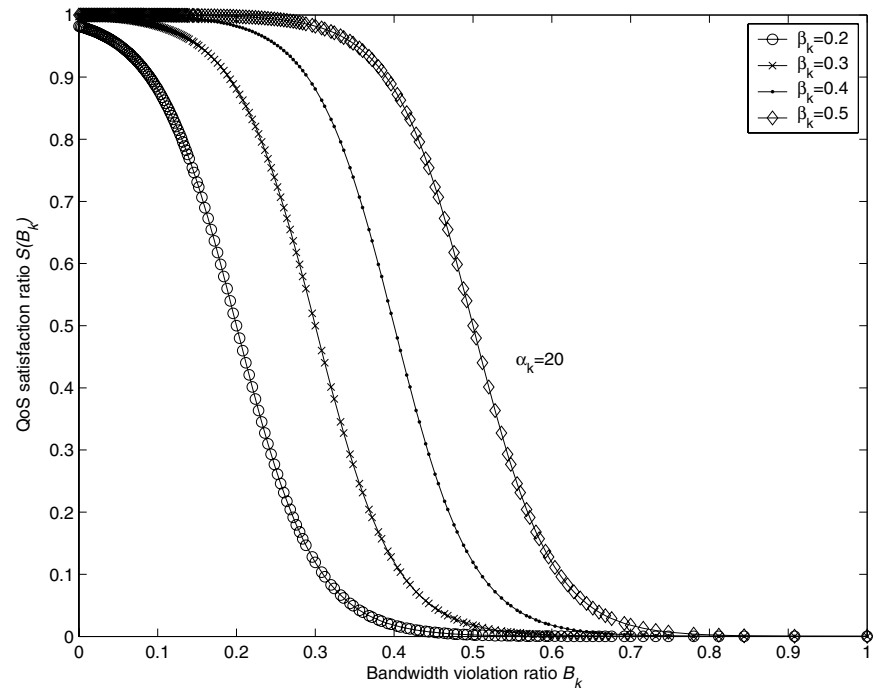

Fig. 2. QoS satisfaction ratio $S\left(B_{k}\right)$ vs. bandwidth violation ratio $B_{k}$ $\left(\beta_{k}=0.2,0.3,0.4,0.5 ; \alpha_{k}=20\right)$.

choose strategy $u_{1}$. Therefore, $q$ will choose $v_{1}$ if $U_{k}-$ $Y_{k}>U_{k}-w O\left(B_{k}\right)$; otherwise $q$ will choose $v_{2}$. The resulting configuration may be $\left\{u_{1}, v_{1}\right\}$ or $\left\{u_{1}, v_{2}\right\}$.

- $R_{k}-L=0$

It is trivial that four configurations are all possible.

- $R_{k}-L<0$

This reasoning process is similar with $R_{k}-L>0$ and the resulting configuration may be $\left\{u_{2}, v_{1}\right\}$ or $\left\{u_{2}, v_{2}\right\}$.

\section{Performance Evaluation}

We simulated the MAC layer protocol based on EDCF. The underlying physical layer we adopt is the IEEE $802.11 \mathrm{~b}$ standard [2] and the channel bit rate is assumed to be $11 \mathrm{Mbps}$ where multi-rate capability is not supported [1]. The values of 
TABLE II

PARAMETERS USED IN THE SIMULATION

\begin{tabular}{lc}
\hline PHY header & $48 \mu \mathrm{s}$ \\
Preamble duration & $144 \mu \mathrm{s}$ \\
Average channel bit rate & $11 \mathrm{Mbps}$ \\
Propagation delay & $1 \mu \mathrm{s}$ \\
$T_{S I F S}$ & $20 \mu \mathrm{s}$ \\
Length of time slot & $10 \mu \mathrm{s}$ \\
MAC header & $272 \mathrm{bits}$ \\
RTS & $160 \mathrm{bits}$ \\
CTS & $112 \mathrm{bits}$ \\
ACK & $112 \mathrm{bits}$ \\
RTSThreshold & $1000 \mathrm{byres}$ \\
\hline \hline$R_{0}, R_{1}, R_{2}$ & $20,10,6$ \\
$L_{0}, L_{1}, L_{2}$ & $10,5,3$ \\
$Y_{0}, Y_{1}, Y_{2}$ & $30,25,15$ \\
$U_{0}, U_{1}, U_{2}$ & $5,5,5$ \\
$V_{0}, V_{1}, V_{2}$ & $0,0,0$ \\
$w$ & 5 \\
\hline
\end{tabular}

TABLE III

TRAFFIC CHARACTERISTICS.

\begin{tabular}{lccc}
\hline & Voice & Video & FTP \\
\hline Codec algorithm & G.729 & H.263 & N/A \\
Average packet inter-arrival time (ms) & 20 & 40 & best effort \\
Payload length (byte) & 20 & 500 & 1500 \\
Bit rate (Kbps) & 8 & 100 & best effort \\
CW $_{\min }$ & 8 & 16 & 32 \\
CWmax & 16 & 32 & 1024 \\
AIFSN & 1 & 1 & 2 \\
Minimum required bandwidth (Kbps) & 8 & 100 & 0 \\
Maximum delay toleration (ms) & 5 & 5 & $\infty$ \\
\hline
\end{tabular}

parameters used in the simulation are summarized in Table II. There are three traffic classes in the simulation: voice, video and FTP. We assume that voice, video and FTP are mapping to traffic class 0 , traffic class 1 and traffic class 2 respectively. The traffic characteristics for these three traffic classes are shown as Table III. It is noted that a FTP flow has no QoS guarantee, i.e., the minimum required bandwidth is zero and the maximum delay toleration is infinite.

The proposed admission control game was simulated to show the performance under an overloading condition, i.e., the system cannot admit any class- $k$ flow to transmit for $0 \leq k \leq 2$. In order to create an overloading environment, the traffic load is continuously offered in the order - one voice flow, one video flow and three FTP flows - until the system cannot admit any class- $k$ flow for $0 \leq k \leq 2$.

For simplicity and convenience, the sensitivities of bandwidth violations and tolerations of bandwidth violations for voice and video flows are assumed to be equal, i.e., $\alpha_{0}=\alpha_{1}$ and $\beta_{0}=\beta_{1}$, and are denote by $\alpha^{*}$ and $\beta^{*}$ respectively. In order to reflect the high toleration of FTP flows, we let
TABLE IV

Number of AdMitTEd Flows $\left(\alpha^{*}=10\right)$.

\begin{tabular}{cccc}
\hline$\beta^{*}$ & $n_{0}$ & $n_{1}$ & $n_{2}$ \\
\hline 0.1 & 6 & 2 & 2 \\
0.2 & 11 & 5 & 6 \\
0.3 & 10 & 8 & 19 \\
0.4 & 10 & 9 & 22 \\
0.5 & 11 & 10 & 22 \\
0.6 & 12 & 11 & 25 \\
0.7 & 13 & 12 & 28 \\
0.8 & 15 & 13 & 31 \\
0.9 & 16 & 14 & 37 \\
1.0 & 17 & 15 & 40 \\
\hline
\end{tabular}

$\alpha_{2}=\infty$ and $\beta_{2}=1$

In Table IV, we show the number of admitted flows in the proposed admission control game, in which the sensitivities of bandwidth violation for voice and video flows are set to 10 , i.e., $\alpha^{*}=10$. In Table IV, we tune the tolerations of bandwidth violations for voice and video flows. It can be seen that as $\beta^{*}$ is greater, the number of admitted flows for each traffic class is greater. System administrators can tune the value of $\beta^{*}$ by considering the tradeoff between QoS guarantees of ongoing flows and the revenue.

In Fig. 3, we show the average throughput for each traffic class in the proposed admission control scheme under different values of sensitivity of bandwidth violations and toleration of bandwidth violations. In Fig. 3(a), the average throughput for voice flows is higher as $\alpha^{*}$ is smaller. Similarly, Fig. 5(b) and Fig. 5(c) which show the average throughput for video flows and FTP flows also have the same situation with Fig. 5(a). As seen from Fig. 5(a), Fig. 5(b), and Fig. 5(c), the average throughput for each traffic is decreasing as $\beta^{*}$ is increasing. The reason is that the number of admitted flows is increasing as $\beta^{*}$ is increasing.

In Fig. 4, we show the average packet delays for different traffic classes under different values of sensitivity of bandwidth violations and toleration of bandwidth violations. The average packet delay for each traffic class is greater as $\beta^{*}$ is greater. Also, the average packet delay for each traffic class is greater as $\alpha^{*}$ is smaller.

Fig. 5 shows the bandwidth violation ratios for voice and video flows. As $\beta^{*}$ is greater, the number of admitted flows is greater and hence the bandwidth violation ratio is greater.

\section{CONCLUSION}

In this paper, we analyzed admission control problem by using game theoretical framework. The system environment we considered was within a local region in which multiple service providers compete for providing wireless Internet services. EDCF protocol was implemented in all APs and mobile stations to provide service differentiation. In the competitive environment, we addressed on the resource management problem that how to increase the revenue for service providers while fulfill most part of QoS satisfactions of ongoing flows.

In the further research, we will consider both real-time and best effort traffic and investigate joint admission and rate 

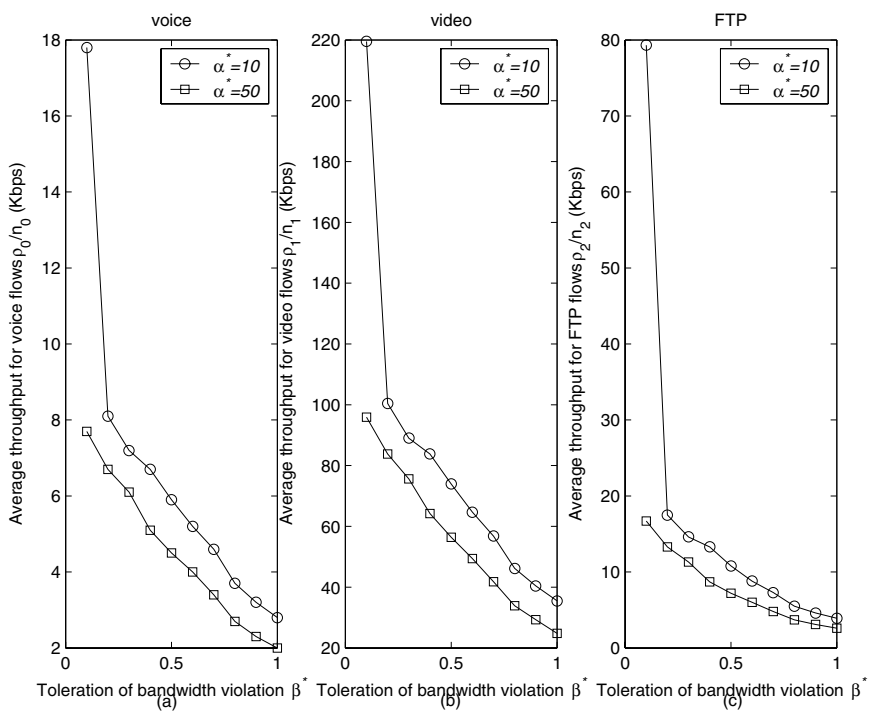

Fig. 3. Average throughput vs. toleration of bandwidth violation. (a) average throughput for voice flows. (b) average throughput for video flows. (c) average throughput for FTP flows.
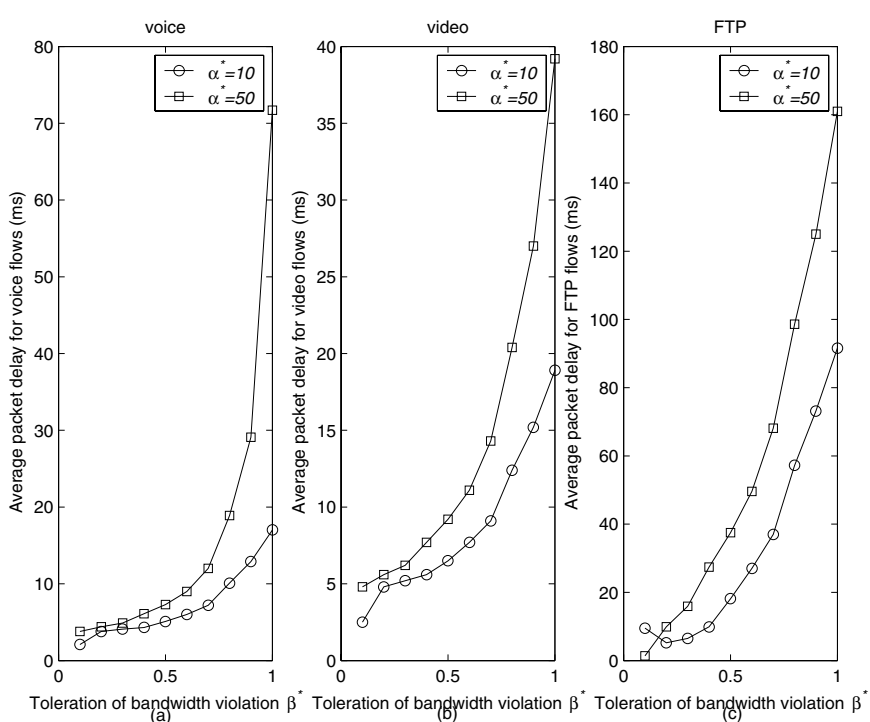

Fig. 4. Average packet delay vs. toleration of bandwidth violation. (a) average packet delay for voice flows. (b) average packet delay for video flows. (c) average packet delay for FTP flows.

controls to not only provide QoS for real-time traffic but also ensure throughput for best effort traffic.

\section{REFERENCES}

[1] Wireless LAN Medium Access Control (MAC) and Physical Layer (PHY) Specifications, IEEE Standard 802.11, 1999.

[2] Wireless LAN Medium Access Control (MAC) and Physical Layer (PHY) Specifications: High-speed Physical Layer Extension in the $2.4 \mathrm{GHz}$ Band, IEEE Standard 802.11b, 1999.

[3] Wireless LAN Medium Access Control (MAC) and Physical Layer (PHY) Specifications:Medium Access Control (MAC) Enhancements for Quality of Service (QoS), IEEE Standard 802.11e/D4.1, February 2003.

[4] T. Basar and G. T. Olsder, Dynamic Noncooperative Game Theory, 2nd Ed., Society of Industrial and Applied Mathematics, 1999.
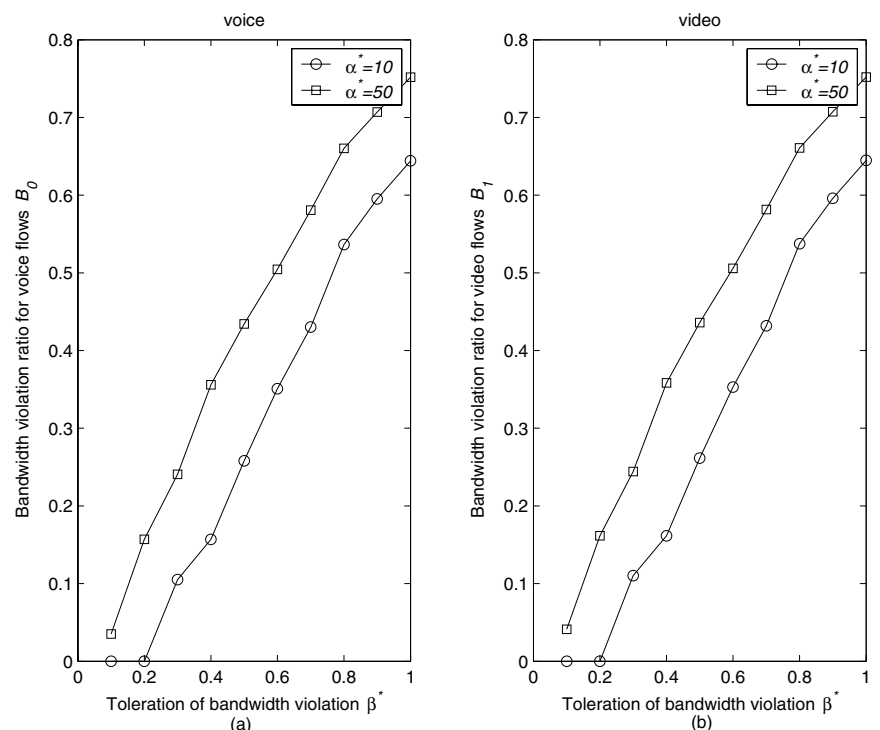

Fig. 5. Bandwidth violation ratio vs. toleration of bandwidth violation. (a) bandwidth violation ratio for voice flows. (b) bandwidth violation ratio for video flows.

[5] D. Gu and J. Zhang, "QoS enhancement in IEEE 802.11 wireless local area networks," IEEE Communication Magazine, vol. 41, pp. 120-124, June 2003.

[6] Y. L. Kuo, C. H. Lu, H. K. Wu, and G. H. Chen, ”An admission control strategy for differentiated services in IEEE 802.11," Proceedings of the IEEE Global Communications Conference (GLOBECOM), vol. 2, San Francisco, December 2003, pp. 707-712.

[7] H. Lin, M. Chatterjee, S. K. Das, and K. Basu, "ARC: An integrated admission and rate control for CDMA Networks based on noncooperative games." Proceedings of ACM International Conference on Mobile Computing and Networking (Mobicom), San Diego, California, Septmber 2003, pp. 326-338.

[8] R. B. Myerson, Game Theory: Analysia of Conflict. Cambridge, MA: Harvard University Press, 1991.

[9] D. Seggern, CRC Standard Curves and Surfaces, Boca Raton, FL, CRC Press, pp. 124, 1993.

[10] U. Varshney, "The status and future of 802.11-based WLANs," Computer, vol. 3, pp. 102-105, June 2003.

[11] M. Xiao, N. B. Shroff, E. K. P. Chong, "Utility-based power control in cellular wireless systems", Proceedings of the IEEE International Conference on Computer Communication (INFOCOM), vol. 1, 2001, pp. $412-421$. 